\title{
Reactivation-Dependent Changes in Memory States in the Terrestrial Slug Limax flavus
}

\author{
Tatsuhiko Sekiguchi, ${ }^{1}$ Atsushi Yamada, and Haruhiko Suzuki
}

Sanyo Electric Co. Ltd.

Tsukuba Research Center

Tsukuba, Ibaraki 305, Japan

\begin{abstract}
The change in memory state in the terrestrial slug Limax flavus was studied using cooling-induced retrograde amnesia. Slugs were first conditioned to avoid carrot odor and then a second conditioning procedure was applied $1,3,6$, or 7 days after the first conditioning trial. Cooling the slugs to $\sim 1^{\circ} \mathrm{C}$ on day 7 immediately after the presentation of the odor used in the conditioning resulted in retrograde amnesia in the slugs that were subject to a second conditioning on day 6 or 7 , but not in slugs that were subject to a second conditioning on day 1 or 3. Next, second-order conditioning was used as the second conditioning procedure to distinguish the memory acquired in the first conditioning from that acquired in the second conditioning and similar results were obtained. These results suggest that the reactivation of memory altered the memory state from a cooling-insensitive state to a cooling-sensitive one. A possible model for memory states is discussed.
\end{abstract}

\section{Introduction}

Animals are evidently capable of storing information regarding a wide variety of events for long periods. After a large number of behavioral experiments, two rather different theoretical frameworks have been developed to explain the way in which long-term storage of information takes place. One is consolidation theory and the other is retrieval theory (Pearce 1987). Memory processes can be

${ }^{1}$ Corresponding author.

E-MAIL t99sekiguchi@tk01.a1.sanyo.co.jp; FAX 81298-372833. divided into three stages-the formation of memory, the storage of information, and the retrieval of memory. Consolidation theories place the greatest emphasis on the first two of these stages. That is, they assume that poor memory is attributable to either inadequate storage because of insufficient rehearsal at the time of training, or to a decay of memory attributable to the passage of time. The concept of short- and long-term memory is implicit in this theory (Duncan 1949; Wagner et al. 1973). On the other hand, retrieval theories (Spear 1973; Lewis 1979) maintain that the formation of memory is very rapid and, once formed, it can be in either an active memory (AM) state or an inactive memory (IM) state. At the time of memory acquisition, information is held in AM, and is coded and elaborated to aid in efficient retrieval. Therefore, according to this theory, poor memory is attributable to inadequate coding or elaboration of the information. After the formation process, the information is stored permanently in IM. External stimuli can trigger the change in the memory state form IM to AM and the formation is then stored after further coding or elaboration.

Experiments on the temporal evolution of memory states have been carried out extensively in a variety of animals, and it is widely accepted that there are at least two time-dependent memory states-short-term memory and long-term memory-irrespective of subject or experimental setup (e.g., see McGaugh 1966; Quinn and Dudai 1976; Menzel 1984; Sekiguchi et al. 1991).

There are only a few reports, however, on the change in the memory state after memory has entered the long-term memory state. Squire and Spanis (1984) reported that in the mouse, the application of electroconvulsive shock induced loss of memory acquired within the 2 weeks before the shock but no loss of memory acquired more than 2 weeks before the shock. Recently, we studied memory states in the terrestrial mollusk, Limax flavus, using cooling-induced retrograde amnesia

LEARNING \& MEMORY 4:356-364 @ 1997 by Cold Spring Harbor Laboratory Press ISSN1072-0502/97 \$5.00

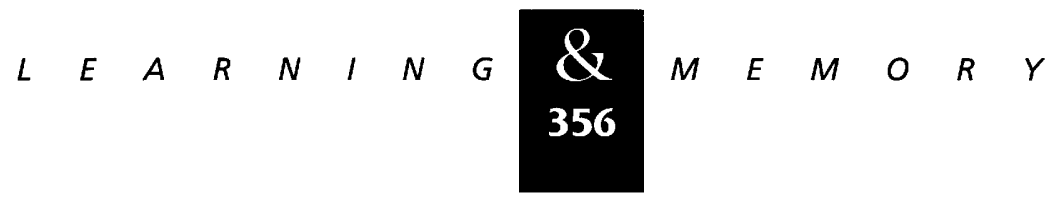


(Yamada et al. 1992), and showed that the acquired memory first entered short-term, then longterm cooling-sensitive and finally cooling-insensitive states. This implies that temporal evolution of the memory state is unidirectional and that the long-term cooling-insensitive state is the final state. To survive in a changing environment, however, animals must change their behavior to adapt to new environment. Therefore, further change in the memory states may be required.

We examined the possibilty of further memory state changes in this study using odor-aversive conditioning of L. flavus. Limax species show a variety of higher-order conditioning effects (Sahley et al. 1981; Gelperin et al. 1985; Sahley et al. 1990) and the memory associations after the conditionings have also been studied (Sahley et al. 1984; Sekiguchi et al. 1994; Suzuki et al. 1994). After the memory acquired during conditioning entered the long-term cooling-insensitive state, the slugs were subjected to additional conditioning [first-order conditioning (FOC) and second-order conditioning (SOC)] and cooling-induced retrograde amnesia was induced to study further alterations in the memory state.

\section{Materials and Methods}

\section{ANIMALS}

The terrestrial mollusk $L$. flavus was raised in the laboratory on frog chow (Oriental Yeast Co. Ltd.) with a 14 -hr/10-hr light/dark cycle at $19^{\circ} \mathrm{C}$. Two or 3 days before the start of training, 2- to 4-month-old animals (1.0-1.5 grams) were placed individually in plastic containers $(113 \times 105 \times 28$ $\mathrm{mm}$ ) lined with moistened filter paper and then starved until the start of the experiment.

\section{MATERIALS USED FOR STIMULATION}

Carrot juice was made in the laboratory by homogenizing several carrots with a blender (100 grams of carrot $/ 100 \mathrm{ml}$ of saline), centrifuging the homogenate for $30 \mathrm{~min}$ at $7000 \mathrm{~g}$, and storing its supernatant at $-20^{\circ} \mathrm{C}$ until use. For the training procedures, carrot juice was applied to filter paper and used as a conditioned stimulus (CS). Cucumber juice was made (100 grams of cucumber $/ 100 \mathrm{ml}$ of saline) and stored in the same manner and used as another CS. A saturated solution (1 gram/90 $\mathrm{ml}$ of saline) of quinidine sulfate was applied to filter paper and used as a bitter-tasting unconditioned stimulus (US). The saline consisted of $52.9 \mathrm{~mm}$ $\mathrm{NaCl}, 4.0 \mathrm{~mm} \mathrm{KCl}, 7.0 \mathrm{~mm} \mathrm{CaCl}_{2}, 4.6 \mathrm{~mm} \mathrm{MgCl}_{2}, 0.2$ $\mathrm{mM} \mathrm{KH}_{2} \mathrm{PO}_{4}, 2.5 \mathrm{~mm} \mathrm{NaHCO}$, and $5.0 \mathrm{~mm}$ dextrose (pH 7.6).

\section{CONDITIONING PROCEDURES}

In every experiment, the slugs were conditioned to avoid carrot and/or cucumber odors by means of the following conditioning procedures.

FOC

The slugs in the conditioned (paired) groups were transferred with forceps to individual plastic containers lined with filter paper moistened with the required CS (carrot or cucumber). After 2 min of exposure, they were transferred with forceps to another plastic container lined with filter paper thoroughly moistened with US (quinidine sulfate) and left for $1 \mathrm{~min}$. The slugs first contacted the quinidine sulfate solution, but soon raised the head to avoid the quinidine sulfate solution and secrete mucus. Then they were washed with saline for 5 sec and returned to their original individual containers. This paired presentation of CS and US was repeated twice with a 2 -hr inter-trial interval. The slugs in the control (unpaired) group in each experiment were subjected to the same number of CS and US presentations as those in the conditioned group, but each CS-US inter-stimulus interval was $30 \mathrm{~min}$.

With the above conditioning oprocedure, slugs were able to sense both odor and taste of CSs. We have already checked that taste-taste conditioning did not contribute significantly to the degree of odor-taste conditioning (see Yamada et al. 1992).

SOC

The second conditioning procedure was applied to slugs that had already been subjected to the FOC procedure described above. After FOC using the first CS (CS1), the slugs in the SOC group were transferred with forceps to individual plastic containers lined with filter paper moistened with the second CS (CS2). After 2 min of exposure, they were transferred to another plastic container lined with filter paper moistened with CS1, with which

$$
\begin{array}{lllllllllllllll}
L & E & A & R & N & I & N & G & \underset{357}{\mathbf{S}} & M & E & M & O & R & Y
\end{array}
$$


they were held in contact for $2 \mathrm{~min}$, then washed with saline for $5 \mathrm{sec}$ and returned to their original individual containers. This paired presentation of CS2 and CS1 was repeated twice with a 2-hr intertrial interval. The slugs in the control (unpaired) group were subjected to the same number of CS2 and CS1 presentations as those in the SOC group, but each CS2-CS1 inter-stimulus interval was 30 min. To counterbalance the two CSs, in half of the slugs in each group, CS1 and CS2 were carrot and cucumber odors, respectively, and in the other half, vice versa.

The details of the experimental procedures and the conditioning cooling, and testing schedules for each experiment are shown in Results or the corresponding figures (Figs. 1-4).

\section{COOLING OF SLUGS}

To induce retrograde amnesia, each slug was exposed to CS1 or CS2 for $2 \mathrm{~min}$, returned to its individual container, and cooled immediately to $-1^{\circ} \mathrm{C}$ in the freezer compartment of a refrigerator. After $5 \mathrm{~min}$ in the freezer, the container with the slug was returned to room temperature, and the slug revived within a few minutes. This cooling procedure was termed "F."

\section{TESTING PROCEDURES}

The testing apparatus has been described elsewhere (Yamada et al. 1992). Briefly, it comprised three chambers, one for carrot or cucumber juice and one for frog chow, as odor sources, which were placed on the floor of each side chamber. The slug was placed in the central chamber, the wall of which was perforated. A line bisected the central chamber into "carrot/cucumber" and "chow" sides.

The testing procedure used was also identical to that described by Yamada et al. (1992). For each trial, a slug was placed in the central chamber with its body aligned along the center line and observed until the head crossed this line. During the next $120 \mathrm{sec}$, the total time during which the slug's head was on the carrot/cucumber side was recorded. In the case of FOC, each slug was subjected to three test trials with carrot odor versus frog chow odor at 2 -hr intervals. In the case of SOC, each slug was subjected to three trials with carrot versus frog chow odor and another three with cucumber versus frog chow odor at 2-hr intervals. The order of carrot or cucumber odor tests was random. The experimenter was unaware of the conditioning treatments experienced by the slug under test.

The conditioning measure used, "odor preference," was defined as the percentage of time the slug spent on the carrot/cucumber side of the chamber in the carrot/cucumber versus frog chow trials. This was obtained by dividing the total amount of time each slug's head was on the carrot/ cucumber side by the total experimental time (120 $\sec \times 3$ trials $=360 \mathrm{sec}$ ) .

It was determined previously that there was no difference in the odor preferences between carrot and cucumber (Sekiguchi et al. 1991).

\section{Results}

\section{TIME-DEPENDENT CHANGES IN MEMORY STATE}

In the first experiment, time-dependent changes in memory state were studied using cooling-induced retrograde amnesia. Slugs were divided into three sets, "cooled," "conditioned," and "control" to compare odor preferences among the sets.

Forty slugs in the cooled set were conditioned to avoid carrot odor using two carrot juice plus quinidine sulfate $(\mathrm{Ca}+\mathrm{Q})$ pairings. Slugs were then given a $\mathrm{Ca}+\mathrm{F}$ treatment on the day of conditioning (group F0, $n=10$ ) or one day (F1, $n=10$ ), three days (F3, $n=10$ ), or 7 days afterward (F7, $n=10$ ). The slugs were tested for carrot odor preference on the day following cooling treatment. The second set of 40 slugs (conditioned set) was divided into four groups (10 slugs/group) and conditioned in the same way as the first set but were not given the cooling treatment. The third set of 40 slugs (control set) was also divided into four groups (10 slugs/group). They were subjected to the control procedure but not cooling treatment. The carrot odor preferences of slugs in the conditioned and control sets were measured on the same day that the odor preference test was administered to the cooled set.

As shown in Figure 1, the carrot odor preference of the slugs in the conditioned set was reduced significantly compared with that of the slugs in the control set and the difference could be observed for at least 7 days after conditioning $(P<0.05)$. This indicated that the conditioning

$$
\text { ….... }
$$



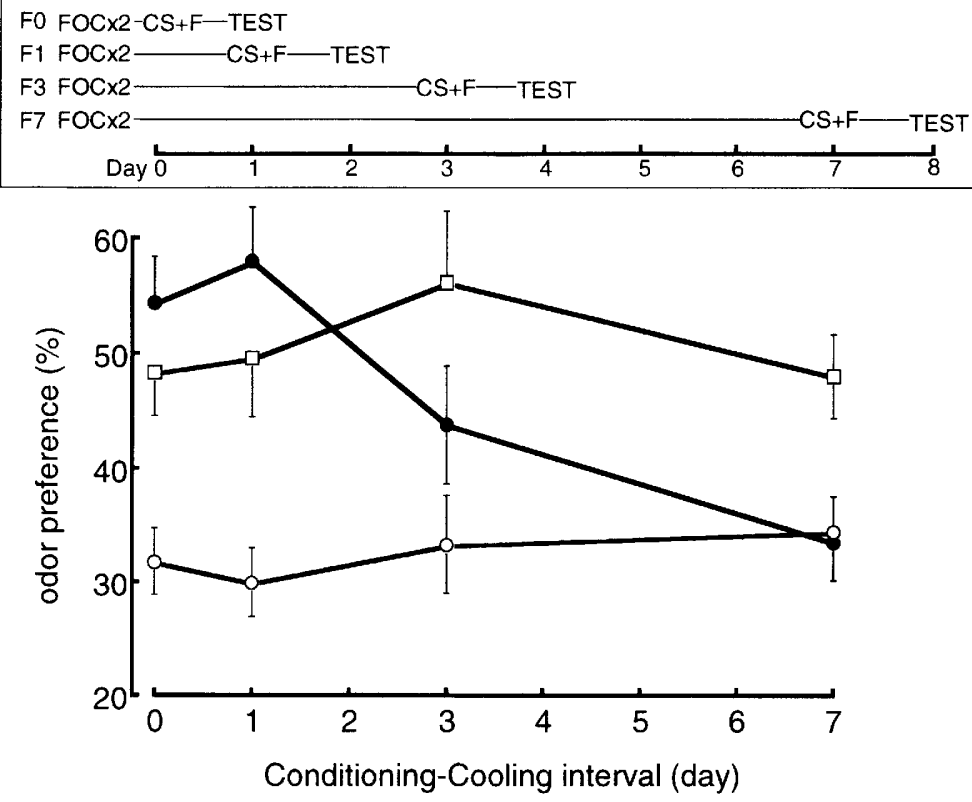

Figure 1: Cooling-induced retrograde amnesia as a function of the conditioning-cooling interval. Slugs in the cooled set were conditioned to avoid carrot odor on day 0 using two carrot juice plus quinidine sulfate $(\mathrm{Ca}+\mathrm{Q})$ conditioning pairs. They were divided into four groups and treated with carrot juice plus cooling procedure $(\mathrm{Ca}+\mathrm{F})$ on the day shown on the abscissa and their carrot odor preference was tested on the next day (O). Another two sets of slugs, FOC (O) and control sets $(\square)$, were subjected to paired and unpaired presentations of two $\mathrm{Ca}+\mathrm{Q}$, respectively, but were not subjected to any cooling treatment. These slugs were tested in the same way as slugs in the cooled set. (Top) Experimental schedule. (Bars) Standard error of the mean. was effective for at least 7 days under our experimental conditions.

Slugs in F0 and F1 groups of cooled set showed carrot odor preference similar to that of control slugs $(P>0.10)$ but a significantly higher carrot odor preference compared with conditioned slugs $(P<0.001)$. This indicated that the cooling-induced retrograde amnesia was induced effectively if $\mathrm{Ca}+\mathrm{F}$ treatment was applied within 1 day after conditioning . The cooling treatment, however, induced retrograde amnesia in slugs less effectively in the F3 group $(0.05<P<0.10)$, and there was no difference between the carrot odor preference of the F7 group and that of the conditioned group $(P>0.8)$. The result was identical with that of our previous study (Yamada et al. 1992) in which the cooling treatment was $\mathrm{Ca}+\mathrm{Q}+\mathrm{F}$ instead of $\mathrm{Ca}+\mathrm{F}$.

Therefore, within a few days after training ( $-3-4$ days), the memory state becomes insensitive to the induction of amnesia.

\section{EFFECTS OF ADDITIONAL TRAINING ON MEMORY STATE}

Forty slugs were conditioned to avoid carrot odor with two $\mathrm{Ca}+\mathrm{Q}$ pairings on day 0 and then were divided into four groups (10 slugs/each group). Each group was given an additional $\mathrm{Ca}+\mathrm{Q}$ training trial on day 1 (group D1), day 3 (D3), day 6 (D6), and day 7 (D7). These slugs were subjected to $\mathrm{Ca}+\mathrm{F}$ treatment on day 7 , and their carrot odor preference was measured on day 8 . Another set of 20 slugs was divided into two groups, FOC $(n=10)$ and control $(n=10)$, to compare their carrot odor preference with that of the slugs described above. Slugs in the FOC and control groups were subjected to three paired and three unpaired presentations of $\mathrm{Ca}+\mathrm{Q}$, respectively, on day $\mathrm{0}$, but were not subjected to any cooling treatment. Their carrot odor preference was tested on day 8 .

The result is shown in Figure 2. As shown in the right-hand columns, the carrot odor preference of FOC slugs was still reduced 8 days after conditioning compared with that of control slugs $[t(18)=3.433, P<0.005]$. This indicated that FOC slugs retained FOC memory 8 days after training.

Slugs in group D1 that were subjected to two $\mathrm{Ca}+\mathrm{Q}$ pairing on day 0 and a third $\mathrm{Ca}+\mathrm{Q}$ pairing on day 1 , followed by $\mathrm{Ca}+\mathrm{F}$ treatment on day 7 , showed reduced carrot odor preference on day 8 $[t(18)=3.467, P<0.005]$. On the other hand, D6 and D7 slugs that were subjected to an additional $\mathrm{Ca}+\mathrm{Q}$ pairing on day 6 or day 7 and $\mathrm{Ca}+\mathrm{F}$ treatment on day 7 showed similar carrot odor preference as that of the controls $[\mathrm{D} 6, t(18)=0.825$, $P>0.4 ; \mathrm{D} 7, t(18)=0.107, P<0.9]$. In addition, groups D6 and D7 showed significantly different preferences from that of group FOC [D6, $t(18)=4.504, \quad P<0.001 ; \quad \mathrm{D} 7, \quad t(18)=3.923$, $P<0.002]$. The preference of group D3 was not different from that of either group FOC or the con-

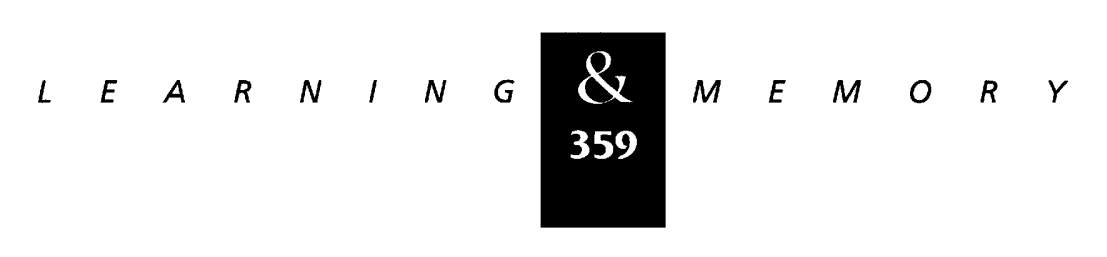



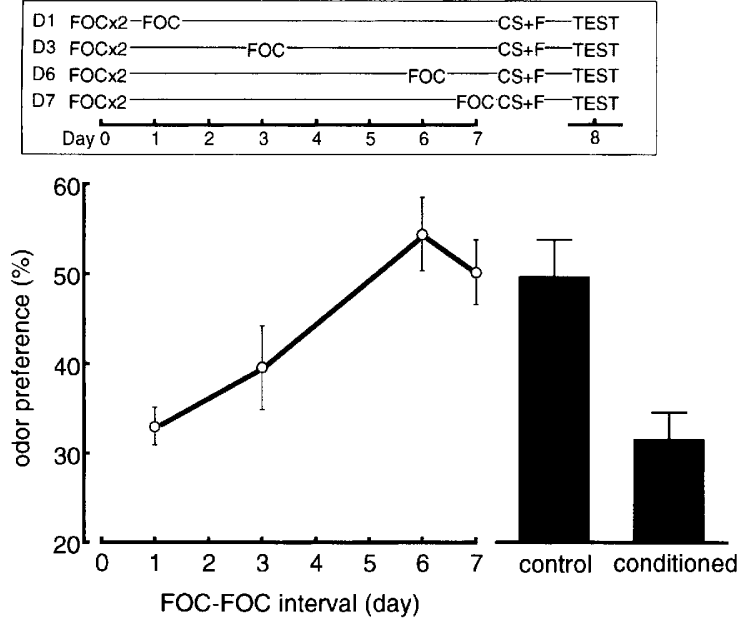

Figure 2: Effect of additional FOC training to the conditioned slugs. Slugs in each group were conditioned to avoid carrot odor on day 0 using two $\mathrm{Ca}+\mathrm{Q}$ conditioning pairs in the same way as described for Fig. 1. Slugs in groups D1, D3, D6, and D7 were subjected to two additional $\mathrm{Ca}+\mathrm{Q}$ pairing on the day shown on the $\mathrm{ab}$ scissa (open circles). All slugs were subjected to $\mathrm{Ca}+\mathrm{F}$ treatment on day 7 and were tested for carrot odor preference on day 8 . Another set of slugs was subjected to paired or unpaired presentations of carrot juice $(\mathrm{Ca})$ and quinidine sulfate $(Q)$, and was tested for carrot odor preference on day 8 (solid bars). (Top) Experimental schedule. (Bars) Standard error of the mean.

trol group $(P>0.10)$. Therefore, $\mathrm{Ca}+\mathrm{F}$ treatment effectively induced retrograde amnesia in D6 and D7 slugs but not in D1 and D3 slugs.

According to Figure 1, on day 7 , memory acquired during FOC training on day 0 should be insensitive to cooling-induced amnesia. D6 and D7 slugs that were subjected to an additional FOC training on D6 and D7, respectively, however, showed increased odor preference compared with that of FOC slugs, which means that $\mathrm{Ca}+\mathrm{F}$ treatment caused amnesia. Therefore, in these slugs, the additional FOC training altered the memory state from a cooling-insensitive to a cooling-sensitive one. As it is diificult in this experiment to distinguish the memory formed by FOC training on day 0 from that formed by the additional training on day 6 or 7 , the following experiment was carried out using a sequential SOC strategy.

\section{EFFECT OF SOC: TREATMENT WITH CS1 + F}

It is well known that there are two types of SOC. One is "sequential SOC," in which CS1 con- ditioned in phase 1 is presented after the presentation of CS2 in phase 2 training procedure. The other is "simultaneous SOC," in which the CS1 conditioned in phase 1 is presented simultaneously with CS2 in phase 2. According to Sekiguchi et al. (1994), cooling treatment to slugs conditioned with those conditioning procedures result in different types of amnesia. In the slugs conditionined with sequential SOC, $\mathrm{CS} 1+\mathrm{F}$ treatment results in increase in only CS1 odor preference and CS2 + F treatment results in increase in both CS1 and CS2 odor preferences. On the other hand, in the slugs conditioned with simultaneous SOC, both CS1 + F and CS2 + F treatments result in increase in both CS1 and CS2 odor preferences. Therefore, it will be better to use sequential SOC to distinguish the memory formed by FOC training on day 0 from that formed by the additional SOC training.

Forty slugs were divided into four groups (10 slugs/each group). On day 0 , all slugs were conditioned to avoid a food odor using two CS1 + US training pairs (FOC). They were then exposed to two CS2 + CS1 training pairs (SOC) on day 1 (group D1), day 3 (D3), day 6 (D6) or day 7 (D7). These slugs were subjected to CS1 + F treatment on day 7 and their odor preferences to CS1 and CS2 were measured on the next day (day 8). Another set of 20 slugs was divided into two groups, SOC $(n=10)$ and control $(n=10)$, to compare their odor preference with that of the slugs described above. Slugs in the SOC group were exposed to two CS1 + US pairing on day 0 and two CS2 + CS1 pairing on day 1 , and those in the control group were subjected to the same number of unpaired presentations. Slugs in these two groups were not subjected to any cooling treatment and their odor preferences to CS1 and CS2 were measured on day 8. As described in Materials and Methods, in half of the slugs in each group (5 slugs), CS1 and CS2 were carrot and cucumber odors, respectively, and in the other half, vice versa, to counterbalance the two CSs. The results shown in Figure 3 represent the combined data of both.

As shown in the right-hand columns in Figure 3 , CS1 and CS2 odor preferences of SOC slugs were still reduced on day 8 compared with those of the control slugs $[\mathrm{CS} 1, t(18)=3.081, P<0.01 ; \mathrm{CS} 2$, $t(18)=2.972, P<0.01]$. This indicated that slugs in SOC group retained SOC memory eight days after the training.

For CS1 odor preference, slugs in group D1, which underwent all the conditioning procedures until day 1 and CS1 + F treatment on day 7 showed

$$
\begin{array}{llllllll}
L & E & A & R & N & I & N & G \\
\begin{array}{l}
\mathcal{Q} \\
360
\end{array} & M & E & M & O & R & Y
\end{array}
$$



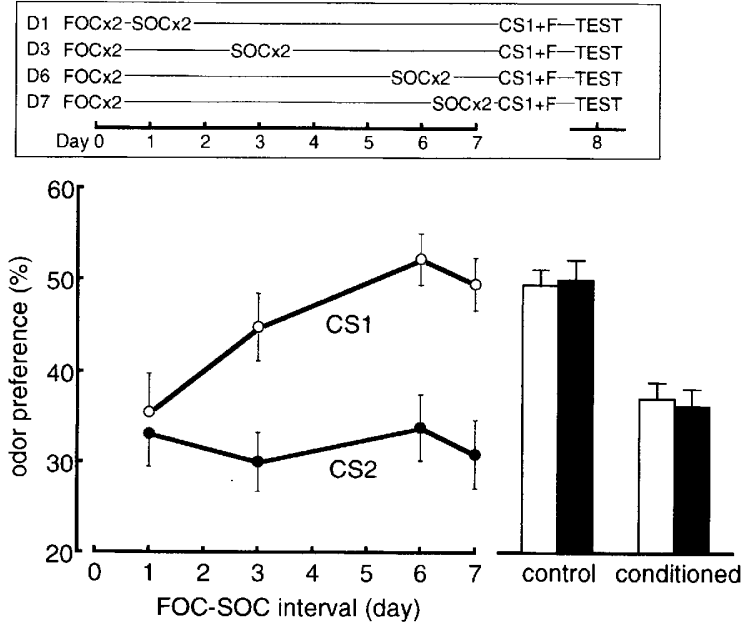

Figure 3: Effect of SOC training to the conditioned slugs - $\mathrm{Ca}+\mathrm{F}$ treatment. Slugs in each group were conditioned to avoid a food odor on day 0 using two CS1 + US conditioning pairs in the same way as described for Fig. 1. Slugs in groups D1, D3, D6, and D7, were subjected to two CS2 + CS1 pairing on the day shown on the abscissa. All slugs were subjected to $\mathrm{CS} 1+\mathrm{F}$ treatment on day 7 and CS1 and CS2 odor preferences were tested on day 8 . Open circles and filled circles show odor preferences to CS1 and CS2, respectively. Another two groups of slugs, SOC and control groups, were subjected to paired and unpaired presentations of $\mathrm{CS} 1+\mathrm{US}$ and $\mathrm{CS} 2+\mathrm{CS} 1$, respectively, and their CS1 and CS2 preferences were tested on day 8 (open columns, CS1; solid bars, CS2). In each group, half of the slugs were conditioned and cooled with carrot juice as CS1 and cucumber juice as CS2, and the other half, vice versa. Combined data are shown. (Top) Experimental schedule. (Bars) Standard error of the mean.

reduced CS1 odor preference $[t(18)=2.719$, $P<0.02]$. On the other hand, slugs in groups D6 and D7, which were subjected to SOC training on days 6 or 7 and CS1 + F treatment on day 7 , showed similar CS1 odor preferences to those of the controls $[\mathrm{D} 6, t(18)=0.696, P>0.40 ; \mathrm{D} 7$, $t(18)=0.021, P<0.9$ ], but significantly different CS1 odor preferences from those of the SOC group $[\mathrm{D} 6, t(18)=3.765, P<0.002 ; \mathrm{D} 7, t(18)=3.081$, $P<0.01]$. The CS1 odor preference of group D3 was not different from that of either the SOC or control groups $(P>0.10)$.

For CS2 odor preference, there were no statistically significant differences among the groups $(P>0.4)$, as expected from our previous finding (Sekiguchi et al. 1994) that CS1 + F treatment did not alter the CS2 odor preference.

Therefore, CS1 + F treatment effectively in- duced retrograde amnesia in D6 and D7 slugs but not in D1 and D3 slugs.

\section{EFFECT OF SOC: TREATMENT WITH CS2 + F}

Forty slugs were divided equally into four groups (10 slugs/each group), D1, D3, D6, and D7. They were subjected to FOC and SOC trainings, cooling treatment and odor preference tests in the same way as shown in Figure 3, except that they were subjected to CS2 + F treatment on day 7 . The CS1 and CS2 odor preferences of each group were compared with those of control and SOC groups of Figure 3.

As shown in Figure 4, slugs in group D1 showed reduced preference to both CS1 and CS2 odors compared with the control group [CS1, $t(18)=3.561, \quad P<0.005 ; \quad$ CS2,$\quad t(18)=3.343$, $P<0.005$ ]. On the other hand, slugs in groups D6 and D7 showed CS1 and CS2 odor preferences similar to those of the control group [for CS1: D6, $t(18)=1.388, P>0.1 ; \mathrm{D} 7, t(18)=0.922, P>0.30$; for CS2: D6, $t(18)=0.12, \quad P>0.90 ; \quad \mathrm{D} 7$, $t(18)=0.371, P>0.50]$. In addition, slugs in groups D6 and D7 were significantly different from
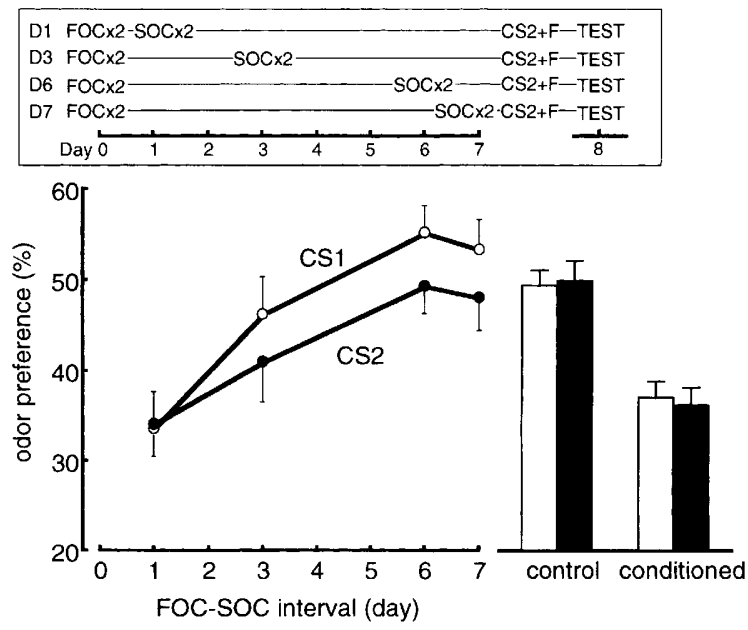

Figure 4: Effect of SOC training to the conditioned slugs-CS2 + F treatment. The grouping of the slugs (D1, D3, D6, and D7) and conditioning, cooling and testing schedules were the same as those for Fig. 3, except that the slugs were subjected to CS2 $+F$ treatment instead of $\mathrm{CS} 1+\mathrm{F}$ on day 7 . In each group, half of the slugs were conditioned and cooled with carrot juice as CS1 and cucumber juice as CS2, and the other half, vice versa. Combined data are shown. Data for SOC and control groups were the same as those in Fig. 3. (Top) Experimental schedule. (Bars) Standard error of the mean.

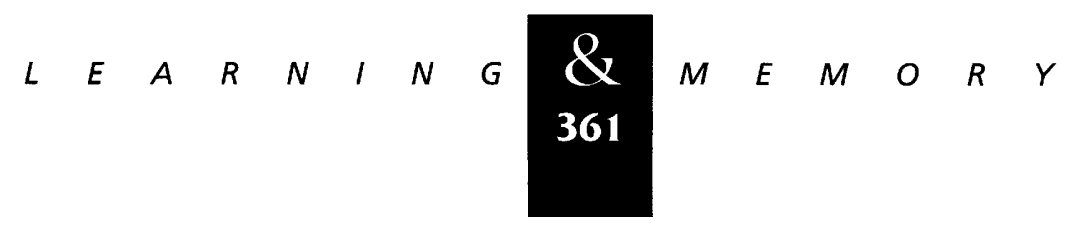


those in the SOC group with respect to both CS1 and CS2 odor preference [for CS1: D6, $t(18)=4.334, \quad P<0.001 ; \quad \mathrm{D} 7, \quad t(18)=3.800$, $P<0.002$; for CS2: D6, $t(18)=2.988, P<0.01 ; \mathrm{D} 7$, $t(18)=2.831, P<0.02$ ] Both CS1 and CS2 odor preferences of slugs in group D3 were not different from those of the slugs in the SOC group or the control group $(P>0.30)$.

As expected from our previous finding (Sekiguchi et al. 1994), CS2 + F treatment affected CS1 and CS2 odor preference in the same way. In fact, no difference was observed between CS1 and CS2 odor preference in any group $(P>0.20)$. Therefore, CS2 + F treatment also retrograde amnesia in slugs in group D6 and D7 but not in slugs in groups D1 and D3, as was the case with CS1 + F treatment.

In Figures 3 and 4, it is clear that SOC training on day 6 or 7 also altered the state of FOC memory from cooling-insensitive to cooling-sensitive.

\section{Discussion}

According to Yamada et al. (1992), FOC memory should be in a cooling-insensitive state on the day of cooling in the experiments illustrated in Figures 2-4. Amnesia was observed in these experiments, however. These results suggest that the memory state was changed from cooling-insensitive to cooling-sensitive by additional FOC or SOC training if this training was applied within a few days before cooling.

In Figure 5, data from Figures 1-4 are combined to show the dependence of odor preference on the interval between the last conditioning treat-

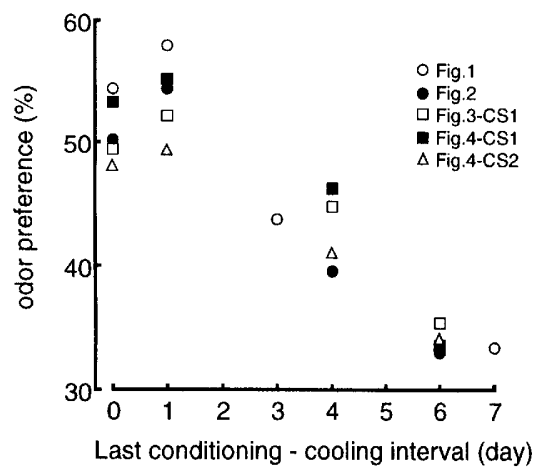

Figure 5: Cooling-induced amnesia as a function of the interval between the last conditioning treatment and cooling. Data (means of odor preferences) from Figs. 1-4 are plotted together, except for CS2 in Fig. 3. ment and cooling. CS2 odor preference in Figure 3 was omitted from this figure because it is reasonable that CS1 + F treatment did not change the CS2 odor preference given the sequential presentation of CS1 and CS2 during phase 2 of training (Sekiguchi et al. 1994). As shown in the figure, the relation of odor preference to the interval was similar in each experiment. This indicated that the time course of evolution memory states remains the same for the memory altered by additional training from cooling-insensitive to cooling-sensitive.

Our previous results showed that temporal evolution of memory state is unidirectional (Yamada et al. 1992). There are at least three states in the memory formation process of the slug-shortterm memory (duration, $\sim 1 \mathrm{~min}$ ), long-term cooling-sensitive memory ( -3 days) and long-term cooling-insensitive memory ( $\sim 1$ month; Sekiguchi et al. 1991). This process can be predicted with reasonable certainty on the basis of the traditional consolidation theory. On the other hand, the present study showed that the memory state is changed easily by new experiences related to the old memory, which is predictable on the basis of the retrieval theory (Lewis 1979; Pearce 1987).

The presence of a reactivated state of memory has been shown by studies in a variety of animals (Misanin et al. 1968; Davis and Klinger 1969; Quinn and Dudai 1975). In the case of Limax, the reactivated memory state was sensitive to cooling as an inducer of retrograde amnesia and had a duration of $\sim 1$ min (Yamada et al. 1992).

These observations, together with the results of the present experiments, suggest the following as a possible model for the memory formation and retrieval process in Limax (Fig. 6). Basically, there are two memory states, cooling-sensitive and cooling-insensitive, each with two substates, active memory (AM) and inactive memory (IM).

\section{MEMORY FORMATION}

Memory itself is formed very rapidly and is cooling-sensitive. At the time of memory acquisition, information is held in AM (corresponding to short-term memory). It is then transferred to IM within $1 \mathrm{~min}$ (long-term memory). Three days later, IM changes from cooling-sensitive to coolinginsensitive. If cooling-insensitive IM is not reactivated within $\sim 1$ month, it will be lost or transformed to a state in which memory retrieval does not occur.

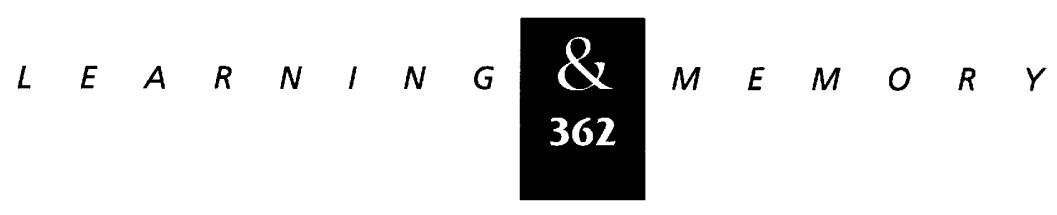




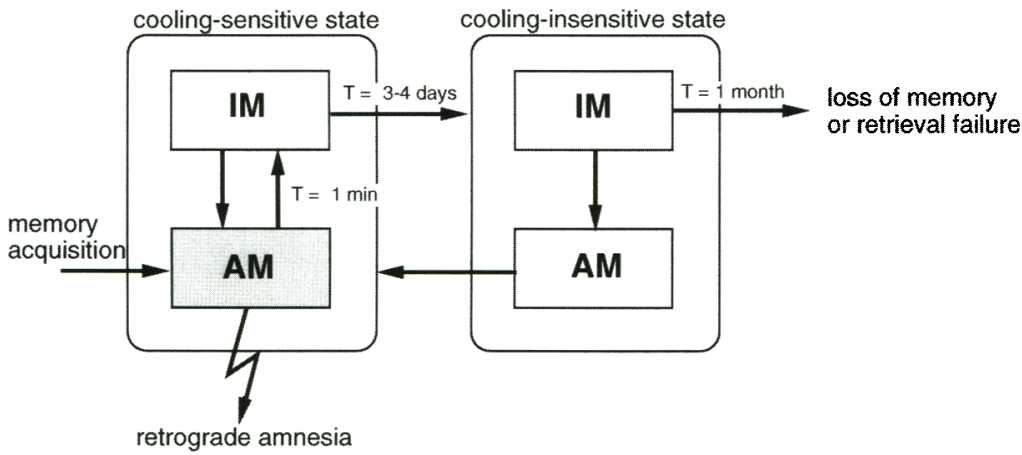

Figure 6: Schematic model of memory state in Limax. The model consists of two memory states, cooling-sensitive and cooling-insensitive states, each with two substates, active memory (AM) and inactive memory (IM). (T) Lifetime of each state. Details are given in the text.
MEMORY RETRIEVAL

External stimuli can trigger the transition from IM to AM (reactivation). The AM reactivated from cooling-sensitive IM is identical to the AM produced in the memory formation process because both memories are sensitive to cooling as an inducer of retrograde amnesia and both have duration of $1 \mathrm{~min}$. In the case of the cooling-insensitive state, there should be AM but amnesia would not be induced by cooling.

\section{LEARNING NEW INFORMATION}

When the animal needs to learn new information related to established memory, external stimuli trigger the transition from IM to AM even in the case of the cooling-insensitive state (memory retrieval), and a new set of information, including memory in the cooling-insensitive state, is held in the cooling-sensitive state.

A part of this model, especially memory formation process of this model, is analogous to that proposed for chick and rats (Allweis 1991) and Drosopbila (Tully 1996). In the case of Drosophila (Tully et al. 1994), memory formation process is divided into five states, information acquired during learning (LRN), short-term memory (STM; decay time $=2 \mathrm{hr}$ ), middle-time memory $(\mathrm{MTM} ; 7 \mathrm{hr})$, anesthesia-resistant memory (ARM; four days), and long-term memory (LTM; >1 week). Although there are differences in the decay times of each memory state, STM, MTM and ARM in Drosophila seem to correspond to our cooling-sensitive AM, cooling-sensitive IM, and cooling-insensitive IM, respectively, from the properties of each memory state. In this study the presence of memory state that corresponded to LTM in Drosopbila was not dissected. But it will be possible to dissect this memory state if conditioning procedure is modi- fied because LTM in Drosopbila is induced only after 10-cycle spaced (15 min) training (Tully et al. 1994).

\section{Acknowledgments}

A part of this study was supported by Special Coordination Funds of the Science and Technology Agency of the Japanese Government.

The publication costs of this article were defrayed in part by payment of page charges. This article must therefore be hereby marked "advertisement" in accordance with 18 USC section 1734 solely to indicate this fact.

\section{References}

Allweis, C. 1991. The congruity of rat and chick multiphasic memory consolidation models. In Neural and behavioral plasticity: The use of the domestic chick as a model (ed. R.J. Andrew), pp. 370-393. Oxford University Press, Oxford, UK.

Davis, R.E. and P.D. Klinger. 1969. Environmental control of amnesic effects of various agents in goldfish. Physiol. Behav. 4: 269-271.

Duncan, C.P. 1949. The retroactive effect of shock on learning. J. Comp. Physiol. Psychol. 42: 32-34.

Gelperin, A., J.J. Hopfield, and D.W. Tank. 1985. The logic of Limax learning. In Model neural networks and behavior (ed. A.I. Selverston), pp. 237-261. Plenum, New York, NY.

Lewis, D.J. 1979. Psychobiology of active and inactive memory. Psychol. Bull. 86: 1054-1083.

McGaugh, J.L. 1966. Time-dependent process in memory storage. Science 153: 1351-1358.

Menzel, R. 1984. Short-term memory in bees. In Primary neural substrates of learning and behavior change (ed. D.L. Alkon and J. Farley), pp. 259-274. Cambridge University Press, Cambridge, MA.

Misanin, J.R., R.R. Miller, and D.J. Lewis. 1968. Retrograde amnesia produced by electroconvulsive shock after reactivation of a consolidated memory trace. Science 160: 554-555.

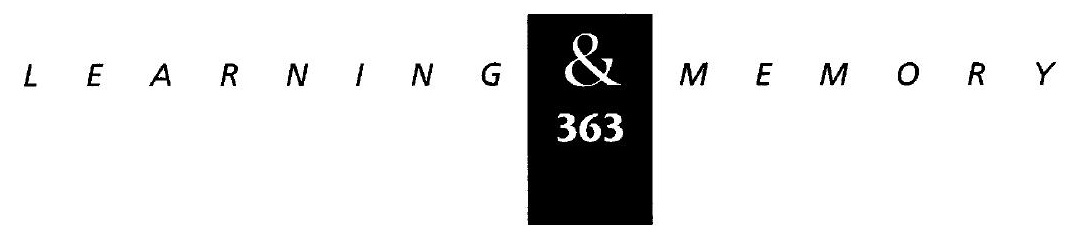




\section{Sekiguchi et al.}

Pearce, J.M. 1987. Introduction to animal cognition. pp. 73-108. Lawrence Erlbaum Associates Ltd., UK.

Quinn, W.G. and Y. Dudai. 1976. Memory phases in Drosophila. Nature 262: 576-577.

Sahley, C.L., K.A. Martin, and A. Gelperin. 1990. An analysis of associative learning in a terrestrial mollusc. II. Appetitive learning. J. Comp. Physiol. A. 167: 339-345.

Sahley, C.L., J.W. Rudy, and A. Gelperin. 1981. An analysis of associative learning in a terrestrial mollusc. 1. High-order conditioning, blocking and a transient US exposure effect. $J$. Comp. Physiol. A. 144: 1-8.

1984. Associative learning in a mollusk: A comparative analysis. In Primary neural substrates of learning and behavior change (ed. D.L. Alkon and J. Farley), pp. 243-258. Cambridge University Press, Cambridge, MA.

Sekiguchi, T., A. Yamada, H. Suzuki, and A. Mizukami. 1991. Temporal analysis of the retention of a food-aversive conditioning in Limax flavus. Zool. Sci. 8: 103-111.

Sekiguchi, T., H. Suzuki, A. Yamada, and A.M. Mizukami. 1994. Cooling-induced retrograde amnesia reflexes Pavlovian conditioning associations in Limax flavus. Neurosci. Res. 18: $267-275$.

Spear, N.E. 1973. Retrieval of memory in animals. Psychol. Rev. 80: 163-194.

Squire, L.R. and C.W. Spanis. 1984. Long gradient of retrograde amnesia in mice: Continuity with the findings in humans. Behav. Neurosci. 98: 345-348.

Suzuki, H., T. Sekiguchi, A. Yamada, and A. Mizukami. 1994. Sensory preconditioning in the terrestrial mollusk, Limax flavus. Zool. Sci. 11: 121-125.

Tully, T. 1996. Discovery of genes involved with learning and memory: An experimental synthesis of Hirschian and Benzerian perspectives. Proc. Natl. Acad. Sci.

93: $13460-13467$.

Tully, T., T. Preat, S.C. Boynton, and M. Del Vecchio. 1994. Genetic dissection of consolidated memory in Drosophila. Cell 79: 35-47.

Wagner, A.R., J.W. Rudy, and J.W. Whitlow. 1973. Rehearsal in animal conditioning. J. Exp. Psychol. 97: 407-426.

Yamada, A., T. Sekiguchi, H. Suzuki, and A. Mizukami. 1992. Behavioral analysis of internal memory states using cooling-insensitive retrograde amnesia in Limax flavus. J. Neurosci. 12: 729-735.

Received April 1, 1997; accepted in revised form September 22, 1997. 


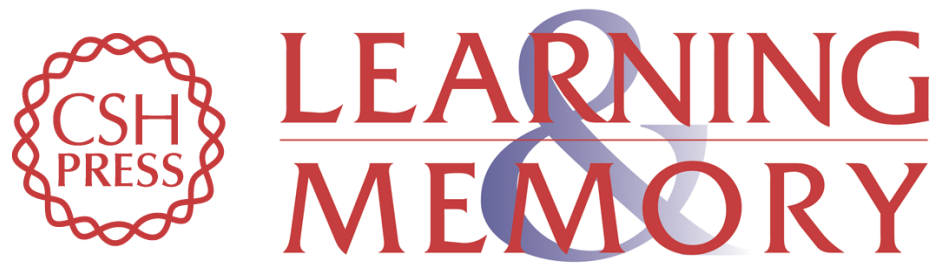

\section{Reactivation-dependent changes in memory states in the terrestrial slug Limax flavus.}

T Sekiguchi, A Yamada and H Suzuki

Learn. Mem. 1997, 4:

Access the most recent version at doi:10.1101//m.4.4.356

References This article cites 17 articles, 4 of which can be accessed free at: http://learnmem.cshlp.org/content/4/4/356.full.html\#ref-list-1

License

Email Alerting Receive free email alerts when new articles cite this article - sign up in the box at the Service top right corner of the article or click here. 